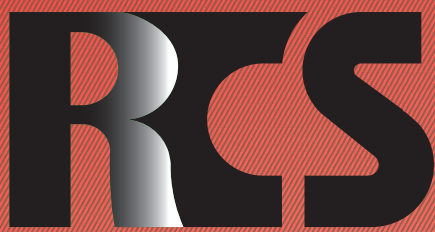

Depósito legal ppi $201502 Z U 4662$

Esta publicación científica en formato digital es continuidad de la revista impresa Depósito Legal: pp $197402 Z$ Z789

- ISSN: 1315-9518 • ISSN-E: 2477-9431

Revista de Ciencias Sociales

Universidad del Zulia. Revista de la Facultad de Ciencias Económicas y Sociales Vol. XXVII,

No. 4,2021

Esta publicación científica en formato digital es continuidad de la revista impresa Depósito Legal: pp $197402 Z 1789$ ISSN: $1315-9518$ 


\title{
Factores críticos de éxito y su impacto en la Gestión de Proyectos empresariales: Una revisión integral
}

\author{
Melendez, Jesus R. \\ El Salous, Ahmed **
}

\section{Resumen}

Actualmente los procesos de gestión empresarial se han focalizado en promover eficientes procedimientos organizacionales basados en métodos tradicionales y marcos agiles de trabajo, junto a la toma de decisiones integrales, las cuales en las primeras fases de la ejecución de los proyectos contribuyen al desarrollo exitoso de sus metas empresariales. Este paper presenta un resumen completo de la literatura reciente sobre los factores principales que inciden en el contexto del desempeño de proyectos dentro de organizaciones empresariales. El objetivo principal se focalizó en identificar los factores involucrados en el desempeño exitoso de la gestión de proyectos. La metodología se basó en el protocolo de una revisión sistémica de literatura con nivel descriptivo. Los resultados se agrupan en factores clave recurrentes involucrados en la gestión exitosa de proyectos, como el tiempo, los costos, la calidad y la capacidad de gestión. Se concluye, que la gestión de proyectos de la mano de sus gerentes deberá focalizarse en nuevos elementos relacionados con el liderazgo, la gestión del conocimiento, estrategias de flexibilidad laboral, ética gerencial, y la moral del equipo de colaboradores para garantizar el éxito de sus operaciones. Estos resultados encontrados podrán ser utilizados como fuente de referencia para futuras investigaciones relacionadas.

Palabras clave: Gestión de proyectos; estrategias; metas empresariales; organizaciones; gerencia.

Ph.D en Gerencia. Magister en Gerencia de las Finanzas y Negocios. Ingeniero Agroindustrial. Profesor y Coordinador de investigación en la carrera de Agroindustria en la Universidad Católica de Santiago de Guayaquil, Ecuador. E-mail: jesus.melendez@cu.ucsg.edu.ec (iD ORCID: https://orcid.org/0000$\underline{0001-8936-5513}$

** $\quad \mathrm{PhD}$ en Proyectos. Master Internacional en Nutrición y Dietética Aplicada. BSc en ciencias, sección de Plantas. Profesor y Director del Instituto de Investigación en la Universidad Agraria del Ecuador, Ecuador. E-mail: eelsalous@uagraria.edu.ec iD ORCID: https://orcid.org/0000-0001-7395-5420

Recibido: 2021-06-01

Aceptado: 2021-08-19 


\title{
Critical success factors and their impact on business Project Management: A comprehensive review
}

\begin{abstract}
Currently, business management processes have focused on promoting efficient organizational procedures based on traditional methods and agile work frameworks, together with comprehensive decisionmaking, which in the early phases of project execution contribute to the successful development of their projects. business goals. This paper presents a comprehensive summary of the recent literature on the main factors influencing the context of project performance within business organizations. The main objective was focused on identifying the factors involved in the successful performance of project management. The methodology was based on the protocol of a systemic literature review with a descriptive level. The results are grouped into key recurring factors involved in successful project management, such as time, costs, quality, and manageability. It is concluded that project management in the hands of its managers should focus on new elements related to leadership, knowledge management, labor flexibility strategies, managerial ethics, and the morale of the team of collaborators to guarantee the success of their operations. These results found may be used as a reference source for future related research.
\end{abstract}

Keywords: Projects management; strategies; business goals; organizations; management.

\section{Introducción}

La dinámica para desarrollar los proyectos comprende una serie de consideraciones en el proceso de planificar los mismos, se destacan el costo, el tiempo, el desempeño técnico y la satisfacción del cliente (Thi y Swierczek, 2010). Estos elementos, son parte del proceso gerencial involucrado en la elaboración de estrategias y planes de control que inciden en el alcance de las metas y del éxito de la organización (Ramos-Soto et al., 2020).

Asimismo, existen factores de éxito involucrados en la gestión del proyecto y en la gerencia del cambio que involucran al personal o empleados de las distintas áreas funcionales o de la operación de los proyectos (Pollack y Algeo, 2016). Esta tendencia organizacional se conecta con la toma de decisiones (Sánchez, Sánchez y Escribá, 2010), y representa un factor determinante en el éxito de las organizaciones dedicadas a la implementación de proyectos.

Existe una complejidad inmersa en la gestión de los proyectos (Spalek, 2014), determinada por la articulación de las estrategias de la organización. Por lo tanto, los eventos resaltantes en la gerencia empresarial se representan por la articulación de procedimientos, factores humanos, problemas externos y las decisiones, las cuales en las primeras fases tienen mayor impacto en la práctica organizacional relacionada con el alcance de objetivos (Alias et al., 2014; Cervantes et al., 2020). En este orden de ideas la planificación del proyecto abarcará el desarrollo de la organización, y control de los procesos junto a factores involucrados con el desempeño óptimo de las actividades que están definidas en cada una de las etapas del ciclo de vida del proyecto.

Los factores definidos como críticos, actúan de forma congruente en el desempeño 

integral

del proyecto, estos abarcan una diversidad compleja de elementos que se sitúan en la motivación del personal para alcanzar los objetivos dentro del cronograma, el presupuesto, la adopción de nuevas tecnologías, criterios de desempeño, y todo un sistema productivo enganchado a los stakeholders (Radujković y Sjekavica, 2017; Vega et al., 2020), los cuales permiten cumplir con el tiempo, costos y la calidad establecida en la gestión de las fases de ejecución del proyecto. Desde esta perspectiva se suma la comunicación, un factor fundamental dentro de las estructuras organizacionales para conectar los distintos procesos, mejorando el rendimiento de los mismos (Hernández, Cogco y Gómez, 2020; Baquero y Cárdenas, 2020).

Por lo tanto, la gestión exitosa de los proyectos empresariales depende de la acertada conjugación de los procesos centrales del negocio, ubicados en el nivel estratégico (Oroujia, 2016), y de la supervisión de los factores considerados como críticos. Este escenario sienta las bases para una gestión del proyecto aplicable en cualquier tipo de organizaciones estructuradas de manera estratégica (Brown, Bocken y Balkenende, 2020), y en correspondencia con las necesidades de mejor rendimiento y maximización en la posibilidad de éxito (Osorio et al., 2014).

En este contexto complejo, se presenta un resumen completo de la literatura reciente sobre los factores críticos que inciden en el desempeño exitoso de proyectos dentro de organizaciones empresariales. Por lo tanto, la pregunta de investigación comprendió: ¿Cuáles son los factores críticos de éxito que son responsables de lograr el éxito en la gestión de proyectos desde un alcance general? El objetivo principal de esta investigación se focalizó en identificar los factores críticos involucrados en el éxito de la gestión de proyectos. La metodología aplicada es del tipo de revisión sistemática de la literatura, con nivel descriptivo. Se consideraron revistas indexadas en las bases de Scopus, WoS, y otras; los resultados se presentan en tablas de contenido a partir de las categorías definidas como, i) Aportes de la gestión de proyectos y su impacto organizacional; y, ii) Complejidad en la Gestión de Proyectos: Perspectivas gerenciales.

\section{Metodología}

Al seguir las recomendaciones de Siddaway, Wood y Hedges (2019), sobre una revisión sistemática de información cualitativa, con nivel exploratorio-descriptivo, se consideraron los procesos de planificación, conducción, organización y presentación, de la meta-síntesis desde los criterios básicos comunes que aportaron los investigadores acerca del fenómeno de estudio relacionado con los factores de éxito involucrados en la gestión de proyectos.

Así, para iniciar la planificación, se formuló la pregunta de investigación en torno a saber ¿Cuáles son los factores críticos de éxito que son responsables de lograr el éxito en la gestión de proyectos desde un alcance general? desde las perspectivas de los investigadores en áreas temáticas a la gestión de proyectos. Esta interrogante condujo la selectividad de los autores y a la propuesta actualizada en función de las categorías: i) Aportes de la gestión de proyectos y su Impacto Organizacional, y ii) Complejidad en la Gestión de Proyectos: Perspectivas gerenciales.

Para recabar la información se consideraron las publicaciones de papers de revistas indexadas de SCOPUS, WoS y otras bases, utilizando estrategias de búsqueda booleana para encontrar desde palabras clave, los papers de interés para esta investigación; esta búsqueda esta definidas por Bramer et al. (2018). La revisión narrativa sistemática, comprendió la formulación de las subcategorías para cada categoría principal las cuales fueron presentadas en tablas de resumen de contenido. La meta-síntesis fue ordenada en cuadros de contenido según lo propuesto por Lachal et al. (2017), estas presentan las subcategorías de mayor dominio descriptivo en las consultas coincidentes, lo cual permitió la triangulación de las fuentes y legitimación 
de los autores.

\section{Aportes de la gestión de proyectos y su impacto organizacional}

Los gerentes de proyecto juegan un importante papel en la dirección exitosa de los proyectos independientemente de la complejidad y del tamaño de sus stakeholders (Jałocha et al., 2014). Los beneficios cuantificables que alcanza la gestión de proyectos de la organización están relacionados con el éxito atribuido a la práctica de inversiones económicas, dentro de la ejecución de las actividades del desarrollo del proyecto, sea industrial, áreas inmobiliarias o de cualquier área de producción de bienes y servicios. De esta manera, si la gestión de los beneficios y la del proyecto se unen, la probabilidad del éxito para la implementación, así como la puesta en marcha de la operación final se ve aumentada en términos del cumplimiento de lo planificado (PapkeShields y Boyer-Wright, 2017) y, por lo tanto, de un mejor desempeño del proyecto y de la organización (Badewi, 2015).

Estas consideraciones son descritas por Zwikael, Chih y Meredith (2018), como una alternativa estratégica que permite establecer beneficios objetivos, basados en costos operativos reducidos, los cuales contribuyen a la mejora a largo plazo del desempeño organizacional luego de la finalización del proyecto, lo cual puede establecer nuevas inversiones del proyecto, una dirección clara de la gestión del mismo y, por lo tanto, un mejor desempeño del proyecto y mayor beneficio para la organización.

Según lo establecido por Gomes y Romão (2016), aquellas organizaciones con estructuras rígidas tradicionalmente burocráticas, no pueden responder de manera efectiva al entorno que exige la implementación de las mejores prácticas de la gerencia empresarial. Es por ello, que la administración de los proyectos incluye varios procesos conjuntos que deben ser gestionados de forma integrada, para garantizar aportes traducidos como beneficios a todos los grupos de interés involucrados, desde los dueños, inversionistas, proveedores, comunidades, y toda la estructura organizacional involucrada.

La gestión de los proyectos actúa sobre la evaluación y control de los indicadores para el dominio del tiempo, de los costos asociados a la operación, y la calidad (Keshk, Maarouf y Annany, 2018), e incluyen además la gestión de riesgos del proyecto, la cual evita muchas pérdidas económicas, físicas, ambientales, al implementar medidas y las normativas legales (Legg et al., 2015). Esta sincronización de procesos, está relacionada con el alcance de metas y objetivos de la organización, en cualquiera de sus fases de desarrollo, así como la puesta en marcha de las operaciones industriales.

Otros beneficios para la organización que desarrollan e implementan proyectos sean de inversión o del tipo social, están determinados por los escenarios donde se desarrolla un diseño de planificación estratégica, conforme a la política general de la organización de proyectos, y que a su vez permitan identificar las oportunidades y riesgos operativos en la gestión de proyectos.

En esta dirección gerencial, se incorporan los procesos de auditorías interna que permiten a la gerencia mejorar los controles internos de la organización, debido a que involucran las operaciones, los informes y el cumplimiento de las metas declaradas en otras áreas de gran importancia como las financieras y administrativas (Chang et al., 2019). Es así como autores como Barros y Oliveira (2017), perfilan la necesidad de evaluar la situación financiera y las condiciones inciertas que pueden afectar el cumplimento de las etapas del proyecto. En este mismo contexto, se incorpora el talento humano disponible para el aporte de conocimientos innovadores, adecuados a las nuevas realidades emergentes y los desafíos del entorno organizacional, que ha de responder a la gestión de proyectos desde la capacidad organizativa.

Otros aportes presentados por Martins y Kunc (2015), establecen que las organizaciones encargadas de desarrollar proyectos, han 

integral

demostrado un comportamiento flexible en la consideración de estrategias y técnicas involucradas en la gestión de proyectos, con el fin de poder conseguir cerrar la brecha entre la planificación y ejecución de la estrategia, así como garantizar a su vez, una mayor efectividad hacia las prácticas orientadas al éxito del proyecto.

Por otra parte, la complementariedad del enfoque de gestión de proyectos conduce al éxito organizacional, al proporcionar una forma flexible de la mejora continua de la calidad y la producción efectiva/eficiente. Los beneficios para la organización se identifican en la estructuración de las actividades, la asignación de los recursos, y los plazos establecidos para guiar los procedimientos en el desempeño de las tareas. Un aspecto significativo tiene que ver con el aprovechamiento de la tecnología y la adaptación al entorno externo para poder impulsar los procesos organizacionales (Boscán y Sandrea, 2020), y orienten los desempeños del talento humano para cumplir con los requisitos, los costos, los riesgos, el tiempo de ejecución, así como la relación con sus stakeholders (Santos y Varajão, 2015; Davis, 2017).

Un factor de éxito adicional en la gestión del proyecto, que se considerada como beneficioso para la organización, lo representa la Sostenibilidad, propuesta por Silvius y Schipper (2014), en el esquema propositivo de la prosperidad del negocio, debido al mantenimiento de prácticas de impacto, en diferentes niveles que comprenden desde la gestión del tiempo, el presupuesto y la calidad; hasta la gestión social, ambiental y económica, así como el reconocimiento de la complejidad, al asumir todos estos escenarios en torno al cambio de mentalidad del gerente del proyecto.

Así, se comprende el interés del conocimiento emergente en la gestión de proyectos, como campo aplicativo de investigación en la idea de predecir los enfoques futuros para las organizaciones. Esto afianzado en los razonamientos de Uchitpe, Uddin y Lynn (2016), quienes destacan que este campo del conocimiento gerencial, es capaz de predecir el futuro desde la aplicabilidad de la gestión de proyectos, frente a la capacidad gerencial bien equipada, lo cual indica que el aprendizaje, la capacidad y el conocimiento puede ayudar a resolver las complicaciones esperadas.

En este orden de ideas, la gestión de proyectos representa en la organización una plataforma accesible para optimizar sus operaciones dentro de los criterios de costos, tiempo y calidad, las cuales se articulan con las experiencias productivas con soporte en la calidad, la participación del empleado en la toma de decisiones de la organización, y su compromiso empresarial, así como en conjunto conectarse a la relación con los stakeholders internos, como el personal de las distintas áreas o procesos de la organización; y los stakeholders externos, como clientes y proveedores. A continuación, se presentan en el Cuadro 1, las principales categorías recurrentes encontradas en la revisión de la literatura. 


\section{Cuadro 1}

\section{Resumen de la categoría: Aportes de la gestión de proyectos y su Impacto Organizacional}

\begin{tabular}{|c|c|c|}
\hline $\begin{array}{c}\text { Subcategorías } \\
\text { Recurrentes }\end{array}$ & $\begin{array}{l}\text { Subcategorías } \\
\text { Recurrentes }\end{array}$ & $\begin{array}{c}\text { Subcategorías } \\
\text { Recurrentes }\end{array}$ \\
\hline $\begin{array}{l}\text { Gestión del tiempo. } \\
\text { Gestión social, ambiental y } \\
\text { económica. } \\
\text { Beneficios para los interesados en el } \\
\text { proyecto. } \\
\text { Superación de brechas entre la } \\
\text { planificación y ejecución de la } \\
\text { estrategia. } \\
\text { Sistema de inversiones. }\end{array}$ & $\begin{array}{l}\text { Toma de decisiones. Capacidad } \\
\text { cognitiva del equipo, costos, } \\
\text { calidad y seguridad. } \\
\text { Coordinación del talento } \\
\text { humano, tiempo, costos, calidad, } \\
\text { seguridad, herramientas y técnicas } \\
\text { innovadoras. }\end{array}$ & $\begin{array}{l}\text { Trabajo de equipo, recursos, ética } \\
\text { del proyecto, control, comunicación, } \\
\text { gestión de riesgos y seguridad. } \\
\text { Participación de las partes interesadas, } \\
\text { aprendizaje organizacional, tiempo, } \\
\text { costo, calidad y sustentabilidad. } \\
\text { Mejora continua y producción } \\
\text { efectiva/eficiente. }\end{array}$ \\
\hline
\end{tabular}

Fuente. Elaboración propia, 2021.

En este contexto complejo, basado en la integración articulada de los procesos definidos en la organización de proyectos, entendiéndose a todos aquellos que forman parte de los niveles estratégicos, funcionales y de operación, pudiéndose citar los administrativos, de planificación estratégica, calidad, ingeniería, procuras, entre otros, se puede identificar una gran participación de elementos clave diferenciados, que son gestionados por los gerentes de proyectos con el fin de establecer controles y mejoras productivas evidenciadas en el cumplimiento de los objetivos planificados.

Este plano de actuación gerencial, permite que la gestión de proyectos se focalice en establecer manejos coordinados y planificados de los tiempos de ejecución de las actividades de cada item programado, al igual que todo un sistema metódico basado en prácticas tradicionales; y en otros casos, mezclas con marcos agiles de trabajo que persiguen la optimización de sus operaciones dentro de los criterios de costos, tiempo y calidad, junto con el compromiso de actuar en equilibrio con sus stakeholders internos y externos.

Estos criterios atraen resultados positivos en el presente y hacia el futuro de la gestión del proyecto en desarrollo, al superar los obstáculos, así como desafíos de equipos, recursos, ética del proyecto, método de control adecuado, eficiente comunicación, y gestión de riesgos (Nusari et al., 2018).

\section{Complejidad en la Gestión de Proyectos: Perspectivas gerenciales}

Los factores y condiciones que se manejan en las organizaciones, a través de la gestión de los proyectos generales, de inversión o de corte social, permiten ampliar la comprensión de procedimientos y enfoques de apoyo para la gestión de proyectos en las distintas ramas de las ciencias de ingeniería, proyectos de construcción de áreas industriales, y no industriales. Asimismo, señalan Yugue y Maximiano (2012), que la complejidad de un proyecto se puede medir en términos de su grado de novedad, sus interdependencias, y las tecnologías involucradas en sus procesos de implementación en la obra (Pérez et al., 2020). En este escenario complejo, las competencias del gerente de proyecto y de sus colaboradores, representan un factor clave para mejorar el desempeño de cada uno de sus procesos organizacionales y su relación con los stakeholders internos o externos (De Moura, Janes y Demoner, 2018).

Algunos autores consideran que la gestión de proyectos, liderada por el gerente y su equipo, es un factor clave para alcanzar el 

integral

éxito del proyecto desde un enfoque integral. Según Armenia et al. (2019), las prácticas corporativas, políticas, recursos, orientación del ciclo de vida, participación de las partes interesadas, aprendizaje organizacional, son metodologías gerenciales, que destacan el creciente interés de proporcionar un impacto en las organizaciones al posicionarse de los factores clave, que pueden generar el mejoramiento interno y externo de la organización desde el punto de vista social, económico, ambiental, manteniendo la combinación óptima de tiempo, costo y desempeño de calidad, de los productos entregados en sus áreas de administrativas, ingeniería, construcción, así como la puesta en marcha de los proyectos.

Las perspectivas gerenciales avanzan hacia la identificación de otros factores considerados como críticos y complejos, y que impactan en el éxito de la gestión de proyectos, mismos que se consolidan en la atención a la sostenibilidad. Las conclusiones presentadas por Koke y Moehler (2019), entregan las bases teóricas de técnicas de gestión para los objetivos de sostenibilidad. Los conceptos de sostenibilidad en sus dimensiones económica, ambiental y social, muestran un valor significativo en los proyectos de construcción (Stanitsas, Kirytopoulos y Leopoulos, 2021), lo cuales conforman un escenario complejo para la gestión, así como avance de los proyectos.

Las propuestas para la medición tradicional del desempeño del proyecto, promueven el método de gestión del valor ganado para rastrear el desempeño de la sostenibilidad en los proyectos sostenibles. Otros hallazgos encontrados y desarrollados por Kivilä, Martinsuo y Vuorinen (2017), establecen que la gestión sostenible de proyectos se implementa utilizando indicadores que abarquen los mecanismos de control para diferentes dimensiones de sostenibilidad, aprovechar las oportunidades de innovación, y así promover la sostenibilidad económica, ambiental y social, como un todo que mejora la rentabilidad general de la organización.

En este sentido práctico, la complejidad del manejo del proyecto centra su interés en otros factores críticos ya mencionados como los criterios de costos, calidad junto a la seguridad, y el manejo de los riesgos, los cuales pueden afectar el éxito del proyecto si no se tiene en cuenta los aspectos de la complejidad administrativa del mismo (San Cristóbal, 2017). Por lo tanto, el éxito de la gestión del proyecto es compleja por las múltiples dimensiones que intervienen en el desarrollo de las actividades planificadas, y se centra en la visión integral de la gerencia (Melendez et al., 2018). Otras dimensiones según Varajão (2016), determinaron que la conducción del proyecto se basaba en tres factores críticos definidos como el alcance, el tiempo y el costo. Estas tres dimensiones y su valoración, indican el grado de eficiencia, así como efectividad de la ejecución del proyecto.

Según la propuesta de San Cristóbal et al. (2018), la perspectiva gerencial puede influir en las formas de abordar la organización para cumplir con la planificación establecida, al tiempo de considerar la interacción con los factores involucrados con una gestión de éxito, estos abarcan la coordinación del talento humano, las experiencias y la selectividad de criterios vinculados al tiempo, costos, calidad, seguridad, y la aplicación de herramientas, técnicas innovadoras, como también sistemas de información e informáticos (Efe y Demirors, 2019), en la estructura global de la red del proyecto.

Los aportes de Vrchota et al. (2021), sobre los factores críticos de éxito en la gestión de proyectos en empresas manufactureras, muestran que las empresas consideran al liderazgo, las experiencias, los empleados y la flexibilidad laboral; mientras que la sostenibilidad de los proyectos, es un factor determinante en las pequeñas y medianas compañías; y finalmente, las empresas del sector de energía llamadas industria 4.0 hacen hincapié en las finanzas como factor importante que decide la implementación de los proyectos.

Otros resultados presentados por Bjorvatn y Wald (2018), destacan que existe una alta correlación entre el éxito del proyecto 
y la flexibilidad como uno de los parámetros del éxito del proyecto. Estos investigadores, presentan el concepto de capacidad de absorción a nivel de equipo, y establecen el papel como mediador entre la complejidad del proyecto y el éxito de la gestión del proyecto. La capacidad de absorción a nivel de equipo, es fundamental para una gestión exitosa del proyecto, pero también que la capacidad de absorción solo puede compensar parcialmente el impacto dañino de la complejidad del proyecto.

En este escenario se retoma el término de las interfaces e interdependencias en la gestión de proyecto. Según Stretton (2016), existe la prioridad de vincular los componentes de un proyecto, a fin de integrarlos desde la mirada sistémica. Los procesos del proyecto no son entidades independientes, puesto que su éxito o el fracaso no está estrictamente bajo el control único del gerente y su equipo del proyecto (Gracia y Melendez, 2019), se consideran otros factores como la falta de apoyo y participación del personal, la definición de objetivos conflictivos, y otros problemas contextuales en el dominio de la alta dirección (Too y Weaver, 2014).

De allí, que los procesos de gestión de los proyectos complejos consideran todo un sistema de interacción organizacional conformado por un canal de comunicación efectivo, miembros del equipo involucrados durante la etapa de diseño, planificación y control de actividades, gestión de riesgos y todas las estrategias comerciales (Ismail, Yusuwan y Baharuddin, 2012). De igual manera, las investigaciones llevadas por $\mathrm{Wu}$ et al. (2017), contribuyen a priorizar un entorno de comunicación dentro de las organizaciones, compuesto por la voluntad de comunicar y la comunicación formal, las cuales se asociaron positivamente con el éxito del proyecto e implementación de los proyectos y la completación de sus etapas.

Otros resultados presentados por la investigación realizada por Gunduz y Yahya (2018), se basaron en identificar y analizar los factores que han estado afectando el éxito, así como el fracaso de los proyectos de construcción; en este sentido, la capacidad técnica de la empresa, el alcance y la definición del trabajo se clasificaron como los factores más importantes. De igual manera otras categorías han sido consideradas, como la complejidad del cronograma, del alcance y la complejidad del costo/recurso (Mirza y Ehsan, 2017), evaluados dentro del proceso de gestión del proyecto.

Tal como señalan Ramlee et al. (2016), la efectividad y la calidad pueden verse afectadas si las escalas de tiempo no se ajustan a los cronogramas establecidos dentro de la planificación del proyecto. Igualmente, la aplicación de metodologías como el Project Management Office (PMO), integran los procesos y mejoran la eficiencia de los gerentes de proyectos (Widforss y Rosqvist, 2015), sin descartar la perspectiva de la gobernanza corporativa (Joslin y Müller, 2016), y del método de acción del gerente, el cual influye en el cumplimiento de la planificación de los demás factores de éxito involucrados en el proyecto (Abubakar et al., 2018). A continuación, se presentan en el Cuadro 2, las principales categorías recurrentes encontradas en la revisión de la literatura. 


\section{Cuadro 2}

\section{Resumen de la categoría: Complejidad en la Gestión de Proyectos: Perspectivas gerenciales}

\begin{tabular}{|c|c|c|}
\hline $\begin{array}{l}\text { Subcategorías } \\
\text { Recurrentes }\end{array}$ & $\begin{array}{l}\text { Subcategorías } \\
\text { Recurrentes }\end{array}$ & $\begin{array}{l}\text { Subcategorías } \\
\text { Recurrentes }\end{array}$ \\
\hline $\begin{array}{l}\text { Competencia técnica de la } \\
\text { organización. Sostenibilidad } \\
\text { evaluación y monitoreo. } \\
\text { Trabajo en equipo, capacidad } \\
\text { gerencial. } \\
\text { Visión de futuro, aprendizaje } \\
\text { y conocimiento. }\end{array}$ & $\begin{array}{l}\text { Gestión de recursos. Ética } \\
\text { del proyecto. Gestión de la } \\
\text { Comunicación. } \\
\text { Relación con stakeholders internos } \\
\text { y externos. } \\
\text { Gestión de riesgos y seguridad. }\end{array}$ & $\begin{array}{l}\text { Participación de las partes interesadas en } \\
\text { los procesos para la toma de decisiones. } \\
\text { Aprendizaje organizacional, tiempo, costo, } \\
\text { calidad y sustentabilidad. } \\
\text { Planificación y gestión de estrategias. }\end{array}$ \\
\hline
\end{tabular}

Fuente. Elaboración propia, 2021.

Estas subcategorías resumidas en el Cuadro 2, determinan la actuación compleja que se establece en la Gestión de los proyectos, basados en la sumatoria de elementos que contemplan una gestión estratégica en todos los procesos involucrados, desde la ejecución de proyectos hasta la entrega de sus productos esperados por el cliente contratante.

La gestión de Proyectos, se presenta como un gran método sistemático personalizado desde las perspectivas de los directores y gerentes, los cuales deben considerar un complejo ambiente, exigidos por los requerimientos del cliente de espacios públicos o privados, junto con otros escenarios basados en otras variables externas en ámbitos sociales, económicos y políticos, así como de forma general con una también compleja interacción con stakeholders internos de la organización, sin descuidar las relaciones con otros grupos de participación externa con la misma, como las sociedades, proveedores, entre otros.

Las investigaciones destacan finalmente que la gestión de proyectos, se ha convertido en una disciplina holística contextual, conductual y fundamentada en la competencia técnica, cuyas tendencias futuras son convincentes y apegadas a la estrategia organizacional (Melendez et al., 2021). Por lo tanto, la perspectiva gerencial sigue avanzando al reconocimiento de los diferentes factores que hacen compleja la ejecución de la práctica de gestión de proyectos, y al mismo tiempo, se establecen medidas para dirigir los factores que favorecen el eficiente desarrollo, como también ejecución de proyectos generales de cualquier tamaño, sean en áreas de emprendimientos y proyectos de pequeñas y medianas empresas, que se enfocan en la sostenibilidad (Vega, Bautista y Martínez, 2020), hasta grandes empresas dedicadas a la construcción (Lima et al., 2021).

\section{Resultados y discusión}

Los resultados obtenidos están determinados por las subcategorías de mayor dominio descriptivo, como son el tiempo, la calidad, el costo y la capacidad gerencial, las cuales han sido encontradas en la selección de los paper analizados; a continuación, se presentan en forma resumida en el Cuadro 3 de análisis de contenido. 


\section{Cuadro 3 \\ Factores críticos recurrentes en la Gestión exitosa de Proyectos}

\begin{tabular}{cc}
\hline $\begin{array}{c}\text { Sub } \\
\text { categorías }\end{array}$ & $\begin{array}{c}\text { Contexto } \\
\text { Organizacional }\end{array}$ \\
\hline
\end{tabular}

Esta subcategoría fue valorada desde el punto de vista de la gestión en el control de las tareas. Los resultados establecen la importancia de los requerimientos del tiempo los cuales Tiempo han de tener holguras en la planificación. La eficiencia en el control se optimiza con el ajuste del cronograma de actividades planificadas en la organización con la ejecución eficiente de las estrategias.

La subcategoría calidad se argumentó por los autores,
como un factor crítico en la gestión de proyectos para la
prosperidad del negocio, por lo tanto, su reconocimiento
gerencial estriba en la mejora continua en el nivel micro de
la organización, lo que significa que es una decisión vital en
el alcance eficiente de los objetivos planificados que asegura
la sustentabilidad en los procesos, considerando los aspectos
sociales, económicos y ambientales.
Esta subcategoría fue recurrente y se direcciona hacia la
evaluación y control de presupuestos y de las inversiones
de forma congruente con la gestión estratégica de las
operaciones de la organización. La evaluación de la
incertidumbre financiera y el ajuste a la calidad de los
procesos están presentes para alcanzar altos desempeños en
la gestión del proyecto.
La manifestación directiva del gerente, se manifiesta en su
Costo
hacer técnico profesional. El desempeño gerencial se precisa
en la aplicación de métodos y técnicas innovadoras, basadas
en sistemas de información gerencial para el beneficio de las
partes interesadas. Los líderes en la gestión de los proyectos
se focalizan en la calidad y reducción de costos los cuales
influyen de manera crítica en el éxito de los proyectos, en un
marco de participación y desarrollo de proyectos sustentables
con el ambiente, y la rentabilidad de la organización.
Adicionalmente se evidencia la focalización en el control
de recursos, capacidades para el apoyo, participación del
personal en la toma de decisiones dentro de la aplicación de
la gestión estratégica.

Procesos

Organizacionales en la eficiencia de los procesos.
Enfoque sistémico. Contexto financiero.

Procesos

Organizacionales y gestión del talento humano.

Fuente. Elaboración propia, 2021.

\section{Conclusiones}

El desarrollo de esta revisión sistémica, partió de la siguiente pregunta de investigación: ¿Cuáles son los factores críticos de éxito que son responsables de lograr el éxito en la gestión de proyectos desde un alcance general? Esta investigación permitió responderla identificando los Factores Críticos de Éxito que se mencionaron con mayor frecuencia en los estudios teóricos revisados y contrastados.

A partir de los resultados este paper presenta tres aportes originales que 

integral

pueden servir como base a investigaciones relacionadas. La primera contribución, es reunir los factores críticos de éxito más citados en la literatura internacional en un solo estudio. El segundo aporte, de este estudio es relacionar los factores críticos de éxito que se encuentran en la literatura con dos categorías relacionadas al aporte de la gestión de proyectos y su impacto organizacional, así como la actuación compleja de la gerencia de proyectos desde una perspectiva gerencial, ambas considerando proyectos generales con descripción de los avances actuales. El tercer aporte significativo de este estudio, es la identificación de los principales factores críticos de éxito que son realmente importantes para explicar el éxito de la gestión de proyectos.

La gestión de proyectos, constituye un componente organizacional de decisiones importantes para cualquier campo del conocimiento. Los resultados establecieron cuatro factores críticos de costo, tiempo, calidad y capacidad gerencial, que deben estar respaldados desde el proceso de la planificación, junto a la aplicación de prácticas adicionales, que permitan abordajes integrales basados en la responsabilidad social corporativa, así como en la sustentabilidad de los proyectos en áreas económicas, sociales y, ambientales.

Esta investigación, aunque presenta un aporte actualizado en el área de gestión de proyectos, no abarca todas las áreas de desarrollo de proyectos de inversión y sociales, por lo que se invita a continuar en otras líneas relacionadas a la gestión individual de proyectos, desde la aplicación de metodologías mixtas, tradicionales y agiles, así como contrastar resultados.

Las futuras investigaciones, deberán abordar la complejidad de elementos organizacionales emergentes, necesarios para visualizar el alcance de los objetivos de la organización, los cuales deberán ser monitoreados en las distintas etapas del proyecto, desde la visualización, conceptualización, el desarrollo de las ingenierías, la implementación, y la etapa de desmantelamiento, según la perspectiva de las partes interesadas. De igual forma, también deberán considerarse el aprendizaje continuo basado en el fortalecimiento de las competencias de los empleados de la organización y del uso de herramientas tecnológicas de la información, las cuales están permitiendo optimizar los resultados exitosos en los proyectos.

\section{Referencias bibliográficas}

Abubakar, A. M., Elrehail, H., Alatailat, M. A., y Elçi, A. (2018). Knowledge management, decision-making style and organizational performance, Journal of Innovation \& Knowledge, 4(2), 104-114. $\quad$ https://doi. org/10.1016/j.jik.2017.07.003

Alias, Z., Zawawi, E. M. A., Yusof, K., y Aris, N. M. (2014). Determining critical success factors of project management practice: A conceptual framework. Procedia - Social and Behavioral Sciences, 153, 61-69. https://doi. org/10.1016/j.sbspro.2014.10.041

Armenia, S., Dangelico, R. M., Nonino, F., y Pompei, A. (2019). Sustainable project management: A conceptualizationoriented review and a framework proposal for future studies. Sustainability, 11(9), 2664. https://doi. org/10.3390/su11092664

Badewi, A. (2015). The impact of project management (PM) and benefits management (BM) practices on project success: Towards developing a project benefits governance framework. International Journal of Project Management, 34(4), 761-778. https://doi.org/10.1016/j. ijproman.2015.05.005

Baquero, D. I., y Cárdenas, S. F. (2020). Redes y actores: Un avance en las relaciones de poder en la comunicación organizacional. Revista 
de Ciencias Sociales (Ve), XXVI(4), 232-245. https://doi.org/10.31876/rcs. v26i4.34660

Barros, A., y Oliveira, S. R.M. (2017). Benefits of using program and project portfolio management on the execution of deliberate and emerging strategies: the accountability perspective. European Journal of Scientific Research, 147(4), 369-377.

Bjorvatn, T., y Wald, A. (2018). Project complexity and team-level absorptive capacity as drivers of project management performance. International Journal of Project Management, 36(6), 876888 . $\quad$ https://doi.org/10.1016/j. ijproman.2018.05.003

Boscán, M., y Sandrea, M. (2020). Cambio estructural para una gestión ecoeficiente: Sector de manufacturas plásticas zulianas en Venezuela. Revista Venezolana de Gerencia, 25(92), 1617-1636. https://doi. org/10.37960/rvg.v25i92.34285

Bramer, W. M., De Jonge, G. B., Rethlefsen, M. L., Mast, F., y Kleijnen, J. (2018). A systematic approach to searching: An efficient and complete method to develop literature searches. Journal of Medical Library Association, 106(4), 531-541. $\quad$ https://doi.org/10.5195/ imla.2018.283

Brown, P., Bocken, N., y Balkenende, R. (2020). How do companies collaborate for circular oriented innovation? Sustainability, 12(4), 1648. https://doi. org/10.3390/su12041648

Cervantes, V., Salgado, R., Peralta, P., y García, C. (2020). Formas organizativas modernas en empresas grandes del sector cooperativo colombiano. Revista de Ciencias Sociales (Ve), XXVI(4), 145-161. https://doi. org/10.31876/rcs.v26i4.34654
Chang, Y-T., Chen, H., Cheng, R. K., y Chi, W. (2019). The impact of internal audit attributes on the effectiveness of internal control over operations and compliance, Journal of Contemporary Accounting and Economics, 15(1), 1-19. $\quad$ https://doi.org/10.1016/j. jcae.2018.11.002

Davis, K. (2017). An empirical investigation into different stakeholder groups perception of project success. International Journal of Project Management, 35(4), 604617. https://doi.org/10.1016/j. ijproman.2017.02.004

De Moura, R. L., Janes, T. C., y Demoner, B. (2018). Influence of the project manager's personal characteristics on project performance. Gestão \& Produção, 25(4), 751-763. https://doi. org/10.1590/0104-530X3595-16

Efe, P., y Demirors, O. (2019). A change management model and its application in software development projects. Computer Standards and Interfaces, 66, 103353. https://doi.org/10.1016/j. csi.2019.04.012

Gomes, J., y Romão, M. (2016). Improving project success: a case study using benefits and project management. Procedia Computer Science, 100, 489-497. https://doi.org/10.1016/j. procs.2016.09.187

Gracia, G. E., y Melendez, J. R. (2019). Challenges of strategic planning in corporate social responsibility: A case of the oil sector. Revista Espacios, 40(27), 2-11.

Gunduz, M., y Yahya, A. M. A. (2018). Analysis of project success factors in construction industry. Technological and Economic Development of Economy, 24(1), 67-80. https://doi.org $/ 10.3846 / 20294913.2015 .1074129$

Hernández, M. D. J., Cogco, A. R., y Gómez, 
M. D. C. (2020). Comunicación de crisis ante la precariedad laboral en las organizaciones. Revista de Ciencias Sociales (Ve), XXVI(4), 115123. https://doi.org/10.31876/rcs. v26i4.34652

Ismail, F., Yusuwan, N. M., y Baharuddin, H. E. A. (2012). Management factors for successful IBS projects implementation. Procedia - Social and Behavioral Sciences, 68, 99107. $\quad$ https://doi.org/10.1016/j. sbspro.2012.12.210

Jałocha, B., Krane, H. P., Ekambaram, A., y Prawelska-Skrzypek, G. (2014). Key competences of public sector project managers. Procedia - Social and Behavioral Sciences, 119, 247-256. https://doi.org/10.1016/j. $\underline{\text { sbspro.2014.03.029 }}$

Joslin, R., y Müller, R. (2016). The impact of project methodologies on project success in different project environments. International Journal of Managing Projects in Business, 9(2), 364-388. https://doi.org/10.1108/ IJMPB-03-2015-0025

Keshk, A. M., Maarouf, I., y Annany, Y. (2018). Special studies in management of construction project risks, risk concept, plan building, risk quantitative and qualitative analysis, risk response strategies. Alexandria Engineering Journal, 57(4), 3179-3187. https://doi. org/10.1016/j.aej.2017.12.003

Kivilä, J., Martinsuo, M., y Vuorinen, L. (2017). Sustainable project management through project control in infrastructure projects. International Journal of Project Management, 35(6), 1167-1183. https://doi.org/10.1016/j. ijproman.2017.02.009

Koke, B., y Moehler, R. C. (2019). Earned Green Value management for project management: A systematic review. Journal of Cleaner Production, 230,
180-197. https://doi.org/10.1016/j. jclepro.2019.05.079

Lachal, J., Revah-Levy, A., Orri, M., y Moro, M. R. (2017). Metasynthesis: An original method to synthesize qualitative literature in psychiatry. Frontiers in Psychiatry. 8, 269. https:// doi.org/10.3389/fpsyt.2017.00269

Legg, S. J., Olsen, K. B., Laird, I. S., y Hasle, P. (2015). Managing safety in small and medium enterprises. Safety Science, 71(PartC). 189-196. https:// doi.org/10.1016/j.ssci.2014.11.007

Lima, L., Trindade, E., Alencar, L., Alencar, M., y Silva, L. (2021). Sustainability in the construction industry: A systematic review of the literature. Journal of Cleaner Production, 289, 125730. https://doi.org/10.1016/j. jclepro.2020.125730

Martins, C. E., y Kunc, M. (2015). Benefits Realisation Management and its influence on project success and on the execution of business strategies. International Journal of Project Management, 33(1), 53-66. https://doi. org/10.1016/j.ijproman.2014.03.011

Melendez, J. R., Pérez, I., García, R., y Piñero, P. (2018). Strategic factors in the context of project management: Management perspectives. Revista Espacios, 39(39), 10-26.

Melendez, J.R., Velasquez-Rivera, J., El Salous, A., y Peñalver, A. (2021). Gestión para la Producción de biocombustibles $2 \mathrm{G}$ : revisión del escenario tecnológico y económico. Revista Venezolana de Gerencia, 26(93), 78-91. https://doi. org/10.52080/rvg93.07

Mirza, E., y Ehsan, N. (2017). Quantification of project execution complexity and its effect on performance of infrastructure development projects. Engineering Management Journal, 29(2), 108-123. https://doi.org/10.1080/10429247.201 


\section{$\underline{7.1309632}$}

Nusari, M., Falasi, M. A., Alrajawy, I., Khalifa, G. S. A., e Isaac, O. (2018). The impact of project management assets and organizational culture on employee performance. International Journal of Management and Human Science, 2(3), 15-26.

Oroujia, M. (2016). Critical success factors in project management. Journal of Project Management, 1, 35-40. https:// doi.org/10.5267/j.jpm.2017.1.001

Osorio, P. C. F., Quelhas. O. L. G., Pérez, L., Shimoda, E., y França, S. (2014). Critical success factors in project management: An exploratory study of an energy company in Brazil. Global Journal of Management and Business Research, XIV(10), 38-50.

Papke-Shields, K. E., y Boyer-Wright, K. M. (2017). Strategic planning characteristics applied to project management. International Journal of Project Management, 35(2), 169-179. https://doi.org/10.1016/j. ijproman.2016.10.015

Pérez, I., García, R., Piñero, P., Saadeq, G., y Peña, M. (2020). Experiencias en el uso de técnicas de softcomputing en la evaluación de proyectos de software. Revista Investigación Operacional, 41(1), 108-119.

Pollack, J., y Algeo, C. (2016). Project managers' and change managers' contribution to success. International Journal of Managing Projects in Business, 9(2), 451-465. https://doi. org/10.1108/IJMPB-09-2015-0085

Radujković, M., y Sjekavica, M. (2017). Project management success factors. Procedia Engineering, 196, 607615. $\quad$ https://doi.org/10.1016/j. proeng.2017.08.048

Ramlee, N., Tammy, N. J., Raja, R. N. H., Musir, A. A., Karin, N. A., Chan, H.
B., y Mohd, S. R. (2016). Critical success factors for construction project. AIP Conference Proceedings, 1774(1), 030011. https://doi. org/10.1063/1.4965067

Ramos-Soto, A. L., Londoño, D. C., Sepulveda-Aguirre, J., y MartínezJiménez, R. (2020). Gestión integral e integrada: Experiencia de las empresas en México. Revista de Ciencias Sociales (Ve), XXVI(3), 31-44. https:// doi.org/10.31876/rcs.v26i3.33229

Sánchez, L., Sánchez, E., y Escribá, A. (2010). Factores determinantes de la intención de cambio estratégico: el papel de los equipos directivos. Cuadernos de Economía y Dirección de la Empresa, (42), 75-112.

San Cristóbal, J. R. (2017). Complexity in project management. Procedia Computer Science, 121, 762766. https://doi.org/10.1016/j. procs.2017.11.098

San Cristóbal, J. R., Carral, L., Díaz, E., Fraguela, J. A., e Iglesias, G. (2018). Complexity and project management: A general overview. Complexity, 2018, 4891286. https://doi. org/10.1155/2018/4891286

Santos, V., y Varajão, J. (2015). PMO as a key ingredient of public sector projects' success - position paper. Procedia Computer Science, 64, 1190-1199. https://doi.org/10.1016/j. procs.2015.08.546

Siddaway, A. P., Wood, A. M., y Hedges, L. V. (2019). How to do a systematic review: A best practice guide for conducting and reporting narrative reviews, metaanalyses, and meta-syntheses. Annual Review of Psychology, 70, 747-770. https://doi.org/10.1146/annurevpsych-010418-102803

Silvius, A. J. G., y Schipper, R. P. J. (2014). Sustainability in project management: 
a literature review and impact analysis. Social Business, 4(1), 6396. https://doi.org/10.1146/annurevpsych-010418-102803

Spalek, S. (2014). Success factors in project management. Literature review. Proceedings of $8^{\text {th }}$ International Technology, Education and Development Conference INTED, 4828-4835. https://doi.org/10.13140/ $\underline{\text { RG.2.1.2439.9127 }}$

Stanitsas, M., Kirytopoulos, K., y Leopoulos, V. (2021). Integrating sustainability indicators into project management: The case of construction industry. Journal of Cleaner Production, 279, 123774. https://doi.org/10.1016/j. jclepro.2020.123774

Stretton, A. (2016). Project interfaces and their management. PM World Journal, $V(7), 1-12$.

Thi, C. H., y Swierczek, F. W. (2010). Critical success factors in project management: implication from Vietnam. Asia Pacific Business Review, 16(4), 567-589. https://doi. org/10.1080/13602380903322957

Too, E. G., y Weaver, P. (2014). The management of project management: a conceptual framework for project governance. International Journal of Project Management, 32(8), 13821394.

Uchitpe, M., Uddin, S., y Lynn, C. (2016). Predicting the future of project management research. Procedia Social and Behavioral Sciences, 226(14), 27-34. https://doi. org/10.1016/j.sbspro.2016.06.158

Varajão, J. (2016). Success management as a PM knowledge area - workin-progress, Procedia Computer Science, 100, 1095-1102. https://doi. org/10.1016/j.procs.2016.09.256

Vega, J. E., Bautista, M. D. C., y Martínez,
M. D. C. (2020). Orientación emprendedora en la innovación de las pequeñas y medianas empresas en México. Revista de Ciencias Sociales (Ve), XXVI(4), 97-114. https://doi. org/10.31876/rcs.v26i4.34651

Vega, V., Ferro, H., Ruiz, M., y Bonomie, M. (2020). Innovación y éxito empresarial: algunas reflexiones teóricas. Revista Venezolana de Gerencia, 25(91), 938953.

Vrchota, J., Řehoř, P., Maříková, M., y Pech, M. (2021). Critical success factors of the project management in relation to industry 4.0 for sustainability of projects. Sustainability, 13(1), 281. https://doi.org/10.3390/su13010281

Widforss, G., y Rosqvist, M. (2015). The project office as project management support in complex environments. Procedia Computer Science, 64, 764-770. https://doi.org/10.1016/j. procs.2015.08.626

Wu, G., Liu, C., Zhao, X., y Zuo, J. (2017). Investigating the relationship between communication-conflict interaction and project success among construction project teams. International Journal of Project Management, 35(8), 14661482. https://doi.org/10.1016/j. ijproman.2017.08.006

Yugue, R. T., y Maximiano, A. C. A. (2012). Project complexity and management processes. Paper presented at PMI ${ }^{\circledR}$ Research and Education Conference, Limerick, Munster, Ireland. https:// www.pmi.org/learning/library/ project-complexity-managementprocesses-6335

Zwikael, O., Chih, Y-Y., y Meredith, J. R. (2018). Project benefit management: Setting effective target benefits. International Journal of Project Management, 36(4), 650658. $\quad$ https://doi.org/10.1016/j. ijproman.2018.01.002 